\title{
CORES, JOINS AND THE FANO-FLOW CONJECTURES
}

\author{
LigAng Jin, ECKHARD SteFFEn \\ Paderborn University \\ Institute of Mathematics and Paderborn Center for Advanced Studies \\ Warburger Str. 100, 33098 Paderborn, Germany \\ e-mail: ligang@mail.upb.de \\ es@upb.de \\ AND \\ Giuseppe Mazzuoccolo \\ Università di Verona \\ Dipartimento di Informatica \\ Strada Le Grazie 15, Verona, Italy \\ e-mail: mazzuoccolo.giuseppe@univr.it
}

\begin{abstract}
The Fan-Raspaud Conjecture states that every bridgeless cubic graph has three 1-factors with empty intersection. A weaker one than this conjecture is that every bridgeless cubic graph has two 1-factors and one join with empty intersection. Both of these two conjectures can be related to conjectures on Fano-flows. In this paper, we show that these two conjectures are equivalent to some statements on cores and weak cores of a bridgeless cubic graph. In particular, we prove that the Fan-Raspaud Conjecture is equivalent to a conjecture proposed in [E. Steffen, 1-factor and cycle covers of cubic graphs, J. Graph Theory 78 (2015) 195-206]. Furthermore, we disprove a conjecture proposed in [G. Mazzuoccolo, New conjectures on perfect matchings in cubic graphs, Electron. Notes Discrete Math. 40 (2013) 235-238] and we propose a new version of it under a stronger connectivity assumption. The weak oddness of a cubic graph $G$ is the minimum number of odd components (i.e., with an odd number of vertices) in the complement of a join of $G$. We obtain an upper bound of weak oddness in terms of weak cores, and thus an upper bound of oddness in terms of cores as a by-product.
\end{abstract}

Keywords: cubic graphs, Fan-Raspaud Conjecture, cores, weak-cores.

2010 Mathematics Subject Classification: 05C70. 


\section{REFERENCES}

[1] G.H. Fan and A. Raspaud, Fulkerson's conjecture and circuit covers, J. Combin. Theory Ser. B 61 (1994) 133-138.

doi:10.1006/jctb.1994.1039

[2] D.R. Fulkerson, Blocking and anti-blocking pairs of polyhedra, Math. Program. 1 (1971) 168-194. doi:10.1007/BF01584085

[3] F. Jaeger, Flows and generalized coloring theorems in graphs, J. Combin. Theory Ser. B 26 (1979) 205-216. doi:10.1016/0095-8956(79)90057-1

[4] L. Jin and E. Steffen, Petersen cores and the oddness of cubic graphs, J. Graph Theory 84 (2017) 109-120. doi:10.1002/jgt.22014

[5] T. Kaiser and A. Raspaud, Perfect matchings with restricted intersection in cubic graphs, European J. Combin. 31 (2010) 1307-1315. doi:10.1016/j.ejc.2009.11.007

[6] D. Král', E. Máčajová, O. Pangrác, A. Raspaud, J.-S. Sereni and M. Škoviera, Projective, affine, and abelian colorings of cubic graphs, European J. Combin. 30 (2009) 53-69. doi:10.1016/j.ejc.2007.11.029

[7] R. Lukot'ka and J. Mazák, Weak oddness as an approximation of oddness and resistance in cubic graphs, arXiv:1602.02949v1 [math.CO] (2016).

[8] E. Máčajová and M. Škoviera, Fano colourings of cubic graphs and the Fulkerson Conjecture, Theoret. Comput. Sci. 349 (2005) 112-120. doi:10.1016/j.tcs.2005.09.034

[9] E. Máčajová and M. Škoviera, Sparsely intersecting perfect matchings in cubic graphs, Combinatorica 34 (2014) 61-94. doi:10.1007/s00493-014-2550-4

[10] G. Mazzuoccolo, New conjectures on perfect matchings in cubic graphs, Electron. Notes Discrete Math. 40 (2013) 235-238. doi:10.1016/j.endm.2013.05.042

[11] E. Steffen, 1-factor and cycle covers of cubic graphs, J. Graph Theory 78 (2015) 195-206. doi:10.1002/jgt.21798

Received 20 January 2016

Revised 24 October 2016

Accepted 26 October 2016 